\title{
Análisis del rendimiento del torneado utilizando coeficiente de vida útil en relación al volumen de metal cortado
}

\author{
Turning performance analysis using coefficient of tool life \\ in relation to the volume of removed material \\ Yoandrys Morales Tamayo ${ }^{1 *} \quad$ Yusimit Zamora Hernández ${ }^{2}$ \\ Patricia del Carmen Zambrano Robledo ${ }^{3} \quad$ Roberto Félix Beltrán Reyna ${ }^{4}$ \\ Recibido 5 de enero de 2016, aceptado 25 de julio de 2016 \\ Received: January 5, 2016 Accepted: July 25, 2016
}

\begin{abstract}
RESUMEN
Los aceros inoxidables austeníticos son materiales difíciles de mecanizar, debido a su alta resistencia, alta ductilidad y baja conductividad térmica. El objetivo de este trabajo es proponer un nuevo criterio para evaluar el rendimiento del torneado de alta velocidad mediante la relación del desgaste del flanco con el volumen de metal cortado. La influencia de los parámetros de corte sobre el coeficiente de vida útil en relación al volumen de metal cortado fue analizada utilizando un análisis de varianza y de regresión múltiple. La investigación mostró un mejor rendimiento del inserto GC2015 en ambas velocidades de corte. El análisis de varianza factorial demostró un efecto significativo del tiempo de mecanizado en el coeficiente de vida útil en relación al volumen de metal cortado.
\end{abstract}

Palabras clave: Torneado de alta velocidad, desgaste del flanco, acero inoxidable AISI 316L, análisis de varianza y regresión, volumen de metal removido.

\begin{abstract}
The austenitic stainless steels are difficult material to machine due to their high strength, high ductility and low thermal conductivity. The aim of this paper is to propose a new criterion for assessing the performance of high speed turning by relating the flank wear with the volume of material removed. The influence of cutting parameters on the coefficient of life in relation to the volume of material removed was analyzed using analysis of variance and multiple regression. The investigation showed a better performance with the GC 2015 insert in both cutting speed. The analysis of factorial variance showed a significant effect of the machinization time in the coefficient of life in relation to the volume of material removed.
\end{abstract}

Keywords: High speed turning, stainless steel AISI 316L, flank wear, variance and regressions analysis, volume of the cut metal.

1 Universidad Técnica de Cotopaxi-Extensión La Maná. Av. Los Almendros y Pujilí S/N, La Maná. Ecuador. E-mail: yoandrys.morales@utc.edu.ec

2 Universidad de Granma. Carretera a Bayamo-Mzllo km 17 1/2. Bayamo. Cuba. E-mail: yzamorah@udg.co.cu

3 Universidad Autónoma de Nuevo León. Pedro de Alba S/N, Ciudad Universitaria, San Nicolás de Los Garza, N.L. México. E-mail: patricia.zambrano@uanl.mx

4 Universidad de las Fuerzas Armadas. Av. General Rumiñahui s/n Ciudad: Sangolquí. País: Ecuador P.O.BOX: 171-5-231B. E-mail:_rfbeltrnr@espe.edu.ec

* Autor de correspondencia 


\section{INTRODUCCIÓN}

Las industrias dedicadas a la manufactura de elementos mecánicos están constantemente esforzándose para reducir costos e incrementar la calidad de las piezas mecanizadas teniendo en cuenta la creciente demanda de productos con una elevada precisión dimensional.

En los últimos años el avance alcanzado en las máquinas herramientas, en las herramientas de corte y en las tecnologías de maquinado han posibilitado la utilización del maquinado de alta velocidad (HSM). Este posibilita una mayor velocidad de remoción de material, reduce los tiempos de maquinado, el número de máquinas herramientas y garantiza un elevado acabado superficial, disminuyendo las fuerzas de corte y la vida útil de la herramienta de corte [1].

La importancia de evaluar el desgaste y predecir la vida de la herramienta radica en los efectos indeseables que provoca, como: una menor exactitud dimensional de la pieza terminada, altas tensiones residuales superficiales, deficiente rugosidad superficial y aumento de las vibraciones durante el proceso de corte [2].

Muchas soluciones para aumentar la vida de la herramienta han estado dirigidas a disminuir la temperatura en la interface herramienta-pieza mediante la utilización y desarrollo de fluidos de corte de diferentes naturalezas. La utilización de estos fluidos encarece los costos de fabricación, provoca daños en la salud de los operarios y afecta el medio ambiente. Las nuevas tecnologías para atenuar los efectos perjudiciales de los fluidos de corte se sustentan en el corte en seco, en la mínima lubricación y en el desarrollo de nuevas fórmulas de fluidos [3].

Actualmente existen numerosas investigaciones que estudian el comportamiento de los aceros austeníticos durante el torneado a altas velocidades. Muchos autores se inclinan por estudiar el desgaste de las herramientas de corte y la rugosidad superficial como principales factores que indican la maquinabilidad del material [4-6]. Otros estudian la integridad superficial [7-8].

Los estudios existentes respecto al desgaste de las herramientas de corte son abordados desde diferentes puntos de vista. Algunos investigadores afirman que el desgaste del flanco es el factor más importante para evaluar el desgaste de la herramienta de corte [9-10].

Otros expresan que en la práctica, es el criterio más usado frecuentemente para determinar la vida útil de la herramienta [11]. Otros enuncian que ha sido el parámetro tradicionalmente más estudiado por encima de los demás tipos de desgaste como es el caso del desgaste del cráter [12].

Ciertos autores consideran este criterio insuficiente para evaluar el rendimiento y desempeño de las herramientas en el proceso de corte y exponen nuevos criterios [13]. Uno de estos autores es Astakhov que en el 2004 propone en una de sus investigaciones varios criterios: el valor de desgaste dimensional, el desgaste relativo superficial, la dimensión específica de la vida útil de la herramienta de corte y el desgaste lineal relativo [14].

En el 2010, Galanis y Manolakos desarrollaron un modelo matemático para predecir la rugosidad superficial en cabezas femorales en el torneado del acero inoxidable AISI 316L. Ellos demostraron que el factor que más afecta la rugosidad superficial es la profundidad de corte seguida por el avance. Señalaron que el mecanizado de alta velocidad se puede usar para fabricar cabezas femorales con rapidez y con calidades superficiales comparadas con los estándares internacionales [15].

Un estudio para comprender el fenómeno de adhesión entre la viruta y la arista de corte de la herramienta fue desarrollo por Gerth y colaboradores [16]. El acero AISI 316L presentó mayor cantidad de material adherido a la superficie en comparación a un acero al carbono.

Por otro, Kara y otros desarrollaron un estudio para predecir la temperatura y las fuerzas de corte durante el torneado ortogonal del acero inoxidable 316L. Los autores expresan que la temperatura de corte aumenta y el radio de formación de la viruta disminuye, debido a la baja conductividad térmica del acero [17].

La presente investigación propone un nuevo criterio que va a relacionar el desgaste del flanco con el volumen de metal cortado. Con este coeficiente se podrá evaluar con efectividad el proceso de torneado, 
teniendo en cuenta que este coeficiente representa el desgaste del flanco de la herramienta de corte por centímetro cúbico de metal cortado.

\section{MATERIALES Y MÉTODOS}

A continuación se muestra el procedimiento ejecutado, el montaje experimental y el diseño de la investigación.

\section{Material de las probetas}

Se utilizó como material para las probetas el acero inoxidable AISI 316L (sin tratamiento térmico) obtenido por los procesos de fundición y laminado en caliente. Es un acero que se toma como patrón típico para la fabricación de productos sometidos a corrosión. Posee bajo porcentaje de carbono y elementos aleantes significativos, lo que favorece su resistencia a la corrosión y sobre todo en presencia de medios ácidos.

Estos aceros son considerados difíciles de mecanizar relacionado fundamentalmente a su baja conductividad térmica, a su alto coeficiente de fricción, alto coeficiente de expansión térmica, alta ductilidad, y un alto endurecimiento por deformación [18]. La composición química del acero AISI 316L es, C $0,015 \%$, Si $0,58 \%$, Mn 1,50\%, Cr 16,95\%, Mo $2,05 \%$, Ni $10,08 \%$, P 0,031\%, S 0,029\% y N 0,059\%.

Herramientas de corte y máquina herramienta Las herramientas de corte utilizadas fueron insertos recubiertos con marcación de Sandvik, PVD GC1115-M15 y CVD GC2015-M15. La geometría seleccionada para los dos insertos fue CCMT 12 04 04-MF con rompevirutas, los insertos fueron depositados en un portaherramientas marca Sandvik de código C6-SCLCL-45065-12 y un adaptador con código C6-391.01-63 060, el ángulo de incidencia principal fue de $7^{\circ}$, el ángulo de ataque fue de $0^{\circ}$ y el radio de la punta fue de $0,4 \mathrm{~mm}$. Se realizó la inspección previa de cada filo de corte para detectar defectos como fragmentos o grietas.

Fue utilizado un torno multifuncional CNC marca tipo Okuma Multus B200-W, con una potencia del motor de $15 \mathrm{~kW}$ y una gama de frecuencia de rotación del husillo que oscila entre 50 y 5.000 $\mathrm{r} / \mathrm{min}$; el peso máximo que soporta sobre el cabezal fijo es de $110 \mathrm{~kg}$.

\section{Montaje experimental y diseño factorial de la investigación}

La investigación consistió en determinar la progresión del coeficiente de vida útil en relación al volumen de metal cortado $\left(C_{u v}\right)$ de dos insertos durante el torneado en seco variando los límites de la velocidad de corte, avance de corte y tiempo principal de corte. Se utilizaron en el estudio diez probetas cilíndricas macizas de diámetro $100 \mathrm{~mm}$ y longitud $200 \mathrm{~mm}$; la relación longitud/diámetro se mantuvo inferior a 10 , para evitar vibraciones durante el mecanizado. La profundidad de corte $(\mathrm{a}=0,3 \mathrm{~mm})$ se mantuvo constante durante el ensayo. En la Tabla 1 aparecen las variables estudiadas.

El experimento fue llevado a cabo utilizando dos niveles de material de la herramienta, dos niveles de velocidad de corte y cuatro niveles de tiempo de mecanizado. Los valores de velocidades de corte fueron seleccionadas superiores a $350 \mathrm{~m} /$ min, rango que según Fernández Abia y otros es considerado de alta velocidad [19]. Los tiempos fueron seleccionados de acuerdo a lo descrito en la norma ASME B94.55M 85 [20].

Se realizaron dos réplicas con cada juego de datos para la adquisición de la información, por lo que en

Tabla 1. Variables consideradas en el estudio.

\begin{tabular}{|l|c|l|l|}
\hline \multicolumn{1}{|c|}{ Variable } & Símbolo & Tipo de variable & \multicolumn{1}{c|}{ Medida } \\
\hline Desgaste de la herramienta & $w(\mu \mathrm{m})$ & Dependiente & $\begin{array}{l}\text { Coeficiente de vida útil en relación al volumen de metal cortado } \\
\left(\mu \mathrm{m} / \mathrm{cm}^{3}\right)\end{array}$ \\
\hline Material de la herramienta & & Independiente & Carburos recubiertos PVD GC1115 y CVD GC2015 \\
\hline Velocidad de corte & $v(\mathrm{~m} / \mathrm{min})$ & Independiente & $400 \mathrm{y} 450 \mathrm{~m} / \mathrm{min}$ \\
\hline Avance de corte & $f(\mathrm{~mm} / \mathrm{rev})$ & Independiente & 0,08 y $0,16 \mathrm{~mm} / \mathrm{rev}$ \\
\hline Tiempo de mecanizado & $T(\mathrm{~min})$ & Independiente & $\begin{array}{l}\text { Tiempo de corte en }(\mathrm{min}) .(2,3,4,5 \mathrm{para} 400 \mathrm{~m} / \mathrm{min}) \mathrm{y}(0,6 ; 1,2 ; \\
2 ; 3 \text { para } 450 \mathrm{~m} / \mathrm{min})\end{array}$ \\
\hline
\end{tabular}


total fueron 64 mediciones. Se ejecutaron ensayos pilotos para comprobar todas las condiciones de experimentación, equipos de medición, máquina herramienta y herramientas de corte.

Se utilizaron los dos filos de corte de cada inserto. Se cilindraron las probetas, midiendo el desgaste del flanco con un microscopio electrónico de barrido marca JEOL. La selección de la probeta, de la herramienta de corte y el orden del ensayo fue completamente aleatoria.

\section{RESULTADOS Y DISCUSIÓN}

La utilización de elevadas velocidades de corte provoca un aumento de la temperatura en la herramienta de corte, como consecuencia debilita la arista de corte [21].

Los insertos maquinados a $400 \mathrm{~m} / \mathrm{min}$ mostraron desgaste por abrasión, adhesión y mueca. Para el caso de la velocidad de $450 \mathrm{~m} / \mathrm{min}$, el inserto GC1115 presenta un excesivo desgaste revelando abrasión, adhesión, difusión, deformación plástica, y fractura del borde cortante, mientras que el GC2015 muestra abrasión, adhesión, difusión, conclusión similar a la obtenida por Rizzuti y Umbrello [22], y Jianxin y otros [23].

Se observó que la herramienta de menor desgaste GC2015 es la de menor dureza, debido a que posee tres recubrimientos específicos para mejorar la mecánica del corte. La capa compuesta por $\mathrm{Ti}(\mathrm{N}, \mathrm{C})$ le proporciona resistencia a el desgaste y estabilidad térmica, la capa de $\mathrm{Al}_{2} \mathrm{O}_{3}$ le suministra resistencia al calor y al desgaste cráter y el recubrimiento de TiN le aporta resistencia al calor y un bajo coeficiente de fricción [24].

Es de mucho interés el estudio del fenómeno de contacto en la superficie del flanco de la herramienta de corte, debido a que su comprensión explica el desgaste del flanco, el que afecta la integridad de la superficie mecanizada. Sin embargo, en los libros modernos no consideran este fenómeno, el desgaste del flanco es estudiado mediante la ecuación de vida útil de Taylor [14].

A continuación se propone un nuevo criterio, el coeficiente de vida útil en relación al volumen de metal cortado $\left(C_{u v}, \mu m / \mathrm{cm}^{3}\right)$, que representa el desgaste del flanco de la herramienta de corte $(w, \mu m)$ por cada $\mathrm{cm}^{3}$ de volumen de metal cortado $\left(V_{o}, \mathrm{~cm}^{3}\right)$, según la ecuación (1).

$$
C_{u v}=\frac{w}{V_{o}}
$$

De esta manera, se relacionan los principales parámetros del régimen de corte con el desgaste del flanco. Este coeficiente permite evaluar desde una nueva arista, la efectividad del proceso de mecanizado por medio de la dependencia entre el desgaste del flanco y el volumen de metal cortado. Resulta conclusivo que la herramienta de corte que logre un menor coeficiente $C_{u v}$, alcanza un mejor desempeño durante la operación de torneado, pues el desgaste disminuye para alcanzar un $\mathrm{cm}^{3}$ de volumen de metal. El volumen de metal cortado $\left(V_{o}\right)$ se determina por la ecuación (2).

$$
V_{o}=v * f * a * T
$$

Donde: $v$ es la velocidad de corte, $f$ es el avance de corte, $a$ es la profundidad de corte y $T$ el tiempo de principal de corte.

En la Figura 1 se muestra la gráfica de $C_{u v}$ para la velocidad de $400 \mathrm{~m} / \mathrm{min}$ se observa un mejor comportamiento al inserto GC1115 hasta los cuatro minutos de corte con un valor de $C_{u v}=1,679 \mu \mathrm{m} /$ $\mathrm{cm}^{3}$, mientras que para el tiempo de cinco minutos el mejor resultado lo alcanzó la plaquita GC2015 con un valor de $C_{u v}=1,604 \mu \mathrm{m} / \mathrm{cm}^{3}$, este comportamiento puede estar motivado a que el inserto GC1115 comienza a perder el recubrimiento y, por tanto, el desgaste crece en mayor proporción en comparación a la otra herramienta de corte.

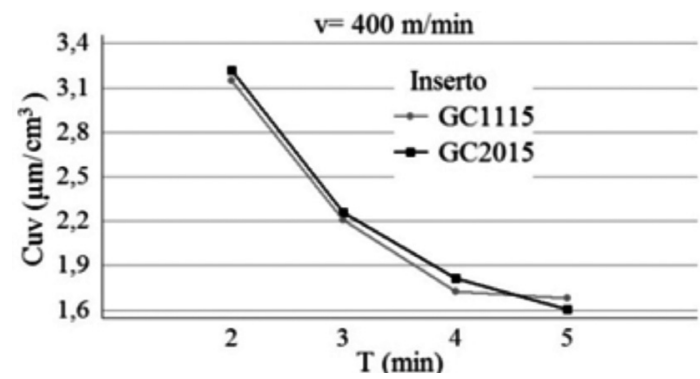

Figura 1. Evolución del coeficiente de vida útil en relación al volumen de metal cortado para $v=400 \mathrm{~m} / \mathrm{min}$. 
Para $v=450 \mathrm{~m} / \mathrm{min}$ (Figura 2)) se puede observar que hasta 1,2 min de corte el inserto GC1115 alcanza los mejores valores de $C_{u v}=5,111 \mu \mathrm{m} /$ $\mathrm{cm}^{3}$, a partir de este tiempo comienza a descender su rendimiento alcanzando un valor máximo para un tiempo de tres minutos de $C_{u v}=14,3535 \mu \mathrm{m} /$ $\mathrm{cm}^{3}$. Este comportamiento se debe al desgaste excesivo que alcanza este inserto perdiendo todo el recubrimiento quedando desprotegido el substrato. Como consecuencia el desgaste crece inversamente proporcional al volumen de metal cortado.

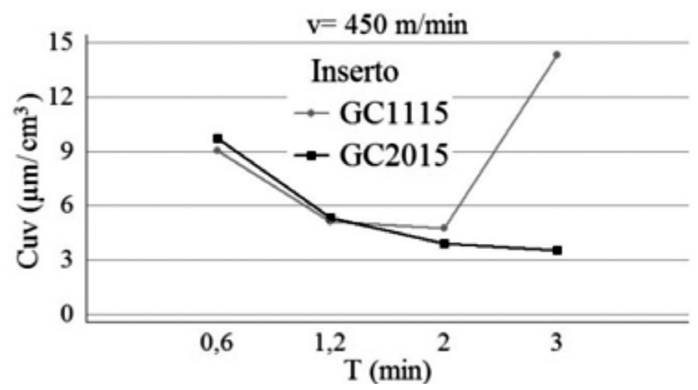

Figura 2. Evolución del coeficiente de vida útil en relación al volumen de metal cortado para $v=450 \mathrm{~m} / \mathrm{min}(\mathrm{b})$.

Para conocer cuáles de las variables influyen de manera significativa en el coeficiente de vida útil en relación al volumen de metal cortado se realiza un análisis de varianza factorial, y luego, teniendo en cuenta esta información se desarrolla un análisis de regresión múltiple con la ayuda del software STATGRAPHICS. Centurion. XV. v15.2.14.

En la Tabla 2 se muestran los resultados de la comparación para las dos velocidades de corte, analizando la contribución de cada factor. Los $p$-valores (probabilidad de $F$ ) reflejan la importancia estadística de cada uno de los factores. El factor tiempo y la interacción avance-tiempo tienen un efecto estadísticamente significativo en $C_{u v}$ para las dos velocidades de corte para un nivel de confianza del $95,0 \%$, ya que el valor de $p$ es inferior a 0,05 .

Sin embargo la variable avance resultó significativa para la velocidad de $400 \mathrm{~m} / \mathrm{min}$, mientras para la velocidad de $450 \mathrm{~m} / \mathrm{min}$ presentan un efecto significativo el factor principal inserto y las interacciones inserto-avance e inserto-tiempo.
Tabla 2. Resultados del análisis de varianza factorial del coeficiente de vida útil en relación al volumen de metal cortado.

\begin{tabular}{|l|c|c|}
\hline $\begin{array}{c}\text { Velocidad } \\
\text { de corte }\end{array}$ & $\boldsymbol{v}=\mathbf{4 0 0} \mathbf{~ m} / \mathbf{m i n}$ & $\boldsymbol{v}=\mathbf{4 5 0} \mathbf{~ m} / \mathbf{m i n}$ \\
\hline Fuente & $p$-valor & $p$-valor \\
\hline Inserto & 0,3510 & 0,0144 \\
\hline Avance & 0,0000 & 0,5507 \\
\hline Tiempo & 0,0000 & 0,0031 \\
\hline Inserto-Avance & 0,7488 & 0,0258 \\
\hline Inserto-Tiempo & 0,3568 & 0,0018 \\
\hline Avance-Tiempo & 0,0000 & 0,0004 \\
\hline
\end{tabular}

Con el objetivo de determinar si al menos una de las medias de $C_{u v}$ es diferente de las demás, se aplica el procedimiento de comparación múltiple. El método utilizado para discernir entre las medias fue el de las menores diferencias significativas (LSD) de Fisher.

Para $v=400 \mathrm{~m} / \mathrm{min}$, la diferencia estimada manifiesta que entre el $C_{u v}$ de los avances $0,08 \mathrm{~mm} / \mathrm{rev}$ y $0,16 \mathrm{~mm} / \mathrm{rev}$, existe una diferencia estadísticamente significativa para un nivel de confianza del $95,0 \%$ siendo el menor valor promedio $1,5306 \mu \mathrm{m} / \mathrm{cm}^{3}$ para el avance $0,16 \mathrm{~mm} / \mathrm{rev}$.

$\mathrm{Al}$ igual que para la variable tiempo todos los grupos alcanzan una diferencia estadísticamente significativa, perteneciendo a un tiempo de dos minutos el menor valor promedio con $1,6419 \mu \mathrm{m} / \mathrm{cm}^{3}$. Por su parte, el gráfico de interacción avance-tiempo revela que el menor de $C_{u v}$ se alcanza con el avance $0,16 \mathrm{~mm} /$ rev para un tiempo de cinco minutos.

Por último, para la velocidad de corte de $450 \mathrm{~m} / \mathrm{min}$, se obtuvo que el $C_{u v}$ de los grupos de los insertos resultaron tener una diferencia estadísticamente significativa para un nivel de confianza del 95,0\%, siendo el menor valor promedio $5,6023 \mu \mathrm{m} / \mathrm{cm}^{3}$ para la plaquita GC2015, mientras que para la variable tiempo fueron significativos los grupos $0,6-1,2$; 0,6-2; 1,2-3 y 2-3 perteneciendo al tiempo de dos minutos el menor valor promedio de 4,3055. Por su parte, los gráficos de las interacciones tiempoinserto, inserto-avance y avance-tiempo revelan que el menor valor de $C_{u v}$ es alcanzado por el avance $0,16 \mathrm{~mm} / \mathrm{rev}$ durante dos minutos de corte.

La regresión múltiple es una técnica estadística que permite determinar la correlación que existe entre 
variables independientes y dos o más variables dependientes. La regresión múltiple se puede utilizar para analizar datos ordinales y categóricos [25]. En este estudio las ecuaciones de regresión fueron determinadas a partir de las medias de los valores de coeficiente de vida útil en relación al volumen de metal cortado bajo las diferentes condiciones de corte planteadas. Las ecuaciones de los modelos ajustados se representan en la Tabla 3.

El proceso de corte es un fenómeno complejo donde convergen muchas variables, es por ello que los investigadores no han podido obtener un modelo que generalice o represente todas las variables. Solo se pueden hacer estudios de caso de manera experimental para describir estos procesos.

Las ecuaciones observadas en la Tabla 3 representan el mejor ajuste de la curva que describen los valores obtenidos de la ecuación 1 bajo las condiciones de corte que se utilizaron en esta investigación.

Con estas ecuaciones se obtuvieron altos valores de coeficientes de determinaciones y pequeños residuos. El coeficiente se ve afectado de diferentes maneras de acuerdo con las condiciones de corte.

Las ecuaciones ofrecen a los operarios e ingenieros de manufacturas un concepto para evaluar la eficiencia del proceso de arranque de viruta teniendo en cuenta el volumen de metal cortado con el desgaste del flanco conociendo el tiempo y el avance de corte para las velocidades de corte utilizadas.

En la Tabla 3 solo se representa una ecuación para la velocidad de $400 \mathrm{~m} / \mathrm{min}$ porque la variable inserto no resulto ser significativa (Tabla 2). El coeficiente de determinación $\left(\mathrm{R}^{2}\right)$ es usado para juzgar el desarrollo adecuado de los modelos de regresión. Un $\mathrm{R}^{2}$ alto indica un mejor ajuste del modelo con los datos experimentales así como un $p$-value cercano a cero refleja la importancia estadística. En esta investigación los modelos alcanzaron valores de $\mathrm{R}^{2}$ mínimos de 88,22 y máximo de 98,02 y los $p$-values en todos los modelos de 0,0000 .

La comparación de las predicciones y los valores experimentales del coeficiente de vida útil en relación al volumen de metal cortado, según el plan factorial completo para las dos velocidades de corte se muestran en las Tablas 4 y 5.

\section{TRABAJOS FUTUROS}

La nueva propuesta del coeficiente estudiado permite evaluar la productividad del mecanizado con la relación de las condiciones de corte fundamentales el desgate del flanco y volumen de metal cortado.

Teniendo en cuenta que es un tema poco estudiado y que se trata de un primer acercamiento a este análisis, se proponen dos líneas de investigación futuras.

- Un análisis profundo de la influencia de las condiciones de corte en el coeficiente de vida útil en relación al volumen de metal cortado.

- Optimización de las condiciones de corte para minimizar el coeficiente de vida útil en relación al volumen de metal cortado.

Tabla 3. Resultado del análisis de regresión del coeficiente de vida útil en relación al volumen de metal cortado.

\begin{tabular}{|c|c|c|c|c|}
\hline Velocidad & Inserto & Ecuación & $\mathbf{R}^{2}$ & $p$-value \\
\hline \multirow{2}{*}{400} & $\begin{array}{l}\mathrm{GC} 1115 \\
\mathrm{GC} 2015\end{array}$ & $C_{u v}=0,357755+\left(\frac{0,614842}{T * f}\right)$ & 98,02 & 0,0000 \\
\hline \multirow{2}{*}{450} & $\mathrm{GC} 1115$ & $C_{u v}=9,94501+0,000388915 * e^{\left(T^{3} * \sqrt{f}\right)}-34,3749 * f$ & 86,49 & 0,0000 \\
\cline { 2 - 6 } & $\mathrm{GC} 2015$ & $C_{u v}=6,45801+14,3722 * \frac{1}{e^{(T+f)}}-34,647 * f$ & 88,22 & 0,0000 \\
\hline
\end{tabular}


Tabla 4. Comparación de la predicciones y los valores experimentales para $v=400 \mathrm{~m} / \mathrm{min}$.

\begin{tabular}{|r|c|c|r|r|r|r|r|}
\hline $\mathbf{N}^{\mathbf{o}}$ & Exp. & Pred. & Residuo & $\mathbf{N}^{\mathbf{o}}$ & Exp. & Pred. & Residuo \\
\hline 1 & 3,9062 & 4,2005 & $-0,2943$ & 17 & 4,2708 & 4,2005 & 0,0703 \\
\hline 2 & 4,3750 & 4,2005 & 0,1745 & 18 & 4,2187 & 4,2005 & 0,0182 \\
\hline 3 & 2,9861 & 2,9196 & 0,0665 & 19 & 2,8819 & 2,9196 & $-0,0377$ \\
\hline 4 & 2,8472 & 2,9196 & $-0,0724$ & 20 & 3,0208 & 2,9196 & 0,1012 \\
\hline 5 & 2,1614 & 2,2791 & $-0,1177$ & 21 & 2,3177 & 2,2791 & 0,0386 \\
\hline 6 & 2,3437 & 2,2791 & 0,0646 & 22 & 2,3697 & 2,2791 & 0,0906 \\
\hline 7 & 2,1041 & 1,8948 & 0,2093 & 23 & 2,1250 & 1,8948 & 0,2302 \\
\hline 8 & 2,1666 & 1,8948 & 0,2718 & 24 & 2,0416 & 1,8948 & 0,1468 \\
\hline 9 & 2,2395 & 2,2791 & $-0,0396$ & 25 & 2,1354 & 2,2791 & $-0,1437$ \\
\hline 10 & 2,0833 & 2,2791 & $-0,1958$ & 26 & 2,2395 & 2,2791 & $-0,0396$ \\
\hline 11 & 1,5104 & 1,6386 & $-0,1282$ & 27 & 1,5625 & 1,6386 & $-0,0761$ \\
\hline 12 & 1,4930 & 1,6386 & $-0,1456$ & 28 & 1,5798 & 1,6386 & $-0,0588$ \\
\hline 13 & 1,1588 & 1,3184 & $-0,1596$ & 29 & 1,2630 & 1,3184 & $-0,0554$ \\
\hline 14 & 1,2239 & 1,3184 & $-0,0945$ & 30 & 1,3020 & 1,3184 & $-0,0164$ \\
\hline 15 & 1,2500 & 1,1263 & 0,1237 & 31 & 1,0833 & 1,1263 & $-0,0430$ \\
\hline 16 & 1,1979 & 1,1263 & 0,0716 & 32 & 1,1666 & 1,1263 & 0,0403 \\
\hline
\end{tabular}

Tabla 5. Comparación de la predicciones y los valores experimentales para $v=450 \mathrm{~m} / \mathrm{min}$.

\begin{tabular}{|r|r|r|r|r|r|r|r|}
\hline \multicolumn{5}{|c|}{ GC1115 } & \multicolumn{4}{c|}{ GC2015 } \\
\hline $\mathbf{N}^{\mathbf{0}}$ & Exp. & Pred. & Residuo & $\mathbf{N}^{\mathbf{0}}$ & Exp. & Pred. & Residuo \\
\hline 1 & 11,8827 & 7,1954 & 4,6873 & 1 & 12,1914 & 10,9679 & 1,2235 \\
\hline 2 & 11,5741 & 7,1954 & 4,3787 & 2 & 12,9630 & 10,9679 & 1,9951 \\
\hline 3 & 6,7129 & 7,1956 & $-0,4827$ & 3 & 6,7901 & 7,6826 & $-0,8925$ \\
\hline 4 & 6,6358 & 7,1956 & $-0,5598$ & 4 & 7,0987 & 7,6826 & $-0,5839$ \\
\hline 5 & 5,9259 & 7,1987 & $-1,2728$ & 5 & 4,9074 & 5,4821 & $-0,5747$ \\
\hline 6 & 6,2963 & 7,1987 & $-0,9024$ & 6 & 4,6759 & 5,4821 & $-0,8062$ \\
\hline 7 & 5,4012 & 8,0012 & $-2,6000$ & 7 & 3,8580 & 4,3472 & $-0,4892$ \\
\hline 8 & 4,7530 & 8,0012 & $-3,2482$ & 8 & 4,4753 & 4,3472 & 0,1281 \\
\hline 9 & 6,1728 & 4,4454 & 1,7274 & 9 & 6,7129 & 7,6367 & $-0,9238$ \\
\hline 10 & 6,4814 & 4,4454 & 2,0360 & 10 & 6,9444 & 7,6367 & $-0,6923$ \\
\hline 11 & 3,5493 & 4,4457 & $-0,8964$ & 11 & 3,6265 & 4,6041 & $-0,9776$ \\
\hline 12 & 3,5493 & 4,4457 & $-0,8964$ & 12 & 3,7037 & 4,6041 & $-0,9004$ \\
\hline 13 & 3,4490 & 4,4545 & $-1,0055$ & 13 & 3,0092 & 2,5728 & 0,4364 \\
\hline 14 & 3,2407 & 4,4545 & $-1,2138$ & 14 & 2,9398 & 2,5728 & 0,3670 \\
\hline 15 & 22,7778 & 23,5099 & $-0,7321$ & 15 & 2,9938 & 1,5250 & 1,4688 \\
\hline 16 & 24,4907 & 23,5099 & 0,9808 & 16 & 2,7469 & 1,5250 & 1,2219 \\
\hline
\end{tabular}

\section{CONCLUSIONES}

De acuerdo con los resultados obtenidos en esta investigación, se plantean las siguientes conclusiones:

- Fue introducido un nuevo criterio para el estudio del rendimiento de la operación de mecanizado, el coeficiente de vida útil en relación al volumen de metal cortado, el que relaciona los principales parámetros de mecanizado con el desgaste del flanco. Este coeficiente permite evaluar desde un nuevo punto de vista, la efectividad del proceso de elaboración por medio de la relación entre el desgaste del flanco y el volumen de metal mecanizado.

- El coeficiente de vida útil en relación al volumen de metal cortado mostró que el inserto GC2015 
alcanzó el menor valor en ambas velocidades estudiadas. Esto significa que para el inserto de tres capas el desgaste del flanco disminuyó en menor proporción al volumen de viruta mecanizado.

- El análisis de varianza factorial demostró que el tiempo de mecanizado tiene un efecto estadísticamente significativo en el coeficiente de vida útil en relación al volumen de metal cortado para un nivel de confianza del $95 \%$. Sin embargo, los demás efectos principales resultaron significativos para una de las velocidades (avance de corte para $400 \mathrm{~m} / \mathrm{min}$ y material inserto para $450 \mathrm{~m} / \mathrm{min}$ ).

- Las ecuaciones de regresión obtenidas en la investigación permiten evaluar el rendimiento y la eficiencia del proceso de mecanizado bajo otras condiciones de corte teniendo en cuenta la relación del desgaste de la herramienta con el volumen del metal mecanizado.

\section{AGRADECIMIENTOS}

Los autores agradecen a la SEP por proporcionar la beca postgraduada de investigación en la Universidad Autónoma de Nuevo León (UANL) en México. Se le agradece al Centro de Investigación e Innovación en Ingeniería Aeronáutica por el apoyo financiero, tecnológico y por todas las facilidades brindadas para el desarrollo del trabajo de investigación.

\section{REFERENCIAS}

[1] L.W. Hernández, R. Pérez, P. Zambrano, M. Guerrero y P. Dumitrescu. "Estudio del desgaste del flanco de carburos recubiertos y cermet durante el torneado de alta velocidad en seco del acero AISI 1045". Revista de Metalurgia. Vol. 47, Issue 3, pp. 262-272. 2011 ISSN: 1988-4222. DOI: 10.3989/ revmetalm.1039.

[2] R. Suresh, S. Basavarajappa y G.L. Samuel. "Some studies on hard turning of AISI 4340 steel using multilayer coated carbide tool". Measurement. Vol. 45, Issue 7, pp. 1872-1884. 2012 ISSN: 0263-2241. DOI: 10.1016/j. measurement.2012.03.024.

[3] S.E. Cordes. "Thermal stability of $\gamma$-alumina PVD coatings and analysis of their performance in machining of austenitic stainless steels". CIRP Journal of Manufacturing Science and Technology. Vol. 5, Issue 1, pp. 20-25. 2012 ISSN: 17555817. DOI: 10.1016/j.cirpj.2011.11.003.

[4] L. Jiang, H. HÄnninen, J. Paro y V. Kauppinen. "Active wear and failure mechanisms of TiN-Coated high speed steel and tin-coated cemented carbide tools when machining powder metallurgically made stainless steels". Metallurgical and Materials Transactions A. Vol. 27, Issue 9, pp. 2796-2808. 1996 ISSN: 1073-5623. DOI: 10.1007/bf02652372.

[5] D. O'Sullivan y M. Cotterell. "Machinability of austenitic stainless steel SS303". Journal of Materials Processing Technology. Vol. 124, Issue 1-2, pp. 153-159. 2002 ISSN: 09240136. DOI: 10.1016/s0924-0136(02)00197-8.

[6] M.A. Xavior y M. Adithan. "Determining the influence of cutting fluids on tool wear and surface roughness during turning of AISI 304 austenitic stainless steel". Journal of Materials Processing Technology. Vol. 209, Issue 2, pp. 900-909. 2009 ISSN: 0924-0136. DOI: 10.1016/j.jmatprotec.2008.02.068.

[7] N. Ben Fredj, H. Sidhom y C. Braham. "Ground surface improvement of the austenitic stainless steel AISI 304 using cryogenic cooling". Surface and Coatings Technology. Vol. 200, Issue 16-17, pp. 48464860. 2006 ISSN: 0257-8972. DOI: 10.1016/j. surfcoat.2005.04.050.

[8] F. Valiorgue, J. Rech, H. Hamdi, P. Gilles y J.M. Bergheau. "3D modeling of residual stresses induced in finish turning of an AISI304L stainless steel". International Journal of Machine Tools and Manufacture. Vol. 53, Issue 1, pp. 77-90. 2012 ISSN: 0890-6955. DOI: 10.1016/j.ijmachtools.2011.09.011.

[9] S. Hasan y S. Thamizhmanii. "Tool flank wear analyses on AISI 440 C martensitic stainless steel by turning". International Journal of Material Forming. Vol. 3, Issue 0, pp. 427-430. 2010 ISSN: 1960-6206. DOI: 10.1007/s12289-010-0798-9.

[10] L. Xihui, Z. Chen, F. Jiwen y G. Song. "A New Method of Tool Wear Measurement". Electrical and Control Engineering (ICECE). Wuhan. 2010.

[11] W. Bouzid Saï. "An investigation of tool wear in high-speed turning of AISI 4340 steel". The International Journal of Advanced Manufacturing Technology. Vol. 26, Issue 4, 
pp. 330-334. 2005 ISSN: 0268-3768. DOI: 10.1007/s00170-003-1991-5.

[12] P. Thangavel, V. Selladurai y R. Shanmugam. "Application of Response Surface Methodology for Predicting Flank Wear in Turning Operation". Proceedings of the Institution of Mechanical Engineers, Part B: Journal of Engineering Manufacture. Vol. 220, Issue 6, pp. 997-1003 2006. DOI: 10.1243/09544054JEM460SC.

[13] H. Zhao, G.C. Barber y Q. Zou. "A study of flank wear in orthogonal cutting with internal cooling". Wear. Vol. 253, Issue 9-10, pp. 957-962. 2002 ISSN: 0043-1648. DOI: 10.1016/s0043-1648(02)00248-x.

[14] V.P. Astakhov. "The assessment of cutting tool wear". International Journal of Machine Tools and Manufacture. Vol. 44, Issue 6, pp. 637-647. 2004.

[15] N. Galanis y D. Manolakos. "Surface roughness prediction in turning of femoral head". The International Journal of Advanced Manufacturing Technology. Vol. 51, Issue 1, pp. 79-86. 2010 ISSN: 0268-3768. DOI: 10.1007/s00170-010-2616-4.

[16] J. Gerth, F. Gustavsson, M. Collin, G. Andersson, L.G. Nordh, J. Heinrichs y U. Wiklund. "Adhesion phenomena in the secondary shear zone in turning of austenitic stainless steel and carbon steel". Journal of Materials Processing Technology. Vol. 214, Issue 8, pp. 1467-1481. 2014 ISSN: 0924-0136. DOI: 10.1016/j. jmatprotec.2014.01.017.

[17] F. Kara, K. Aslantaş y A. Çiçek. "Prediction of cutting temperature in orthogonal machining of AISI 316L using artificial neural network". Applied Soft Computing. Vol. 38, pp. 64-74. 2016 ISSN: 1568-4946. DOI: 10.1016/j. asoc.2015.09.034.

[18] İ. Asiltürk y S. Neşeli. "Multi response optimisation of CNC turning parameters via Taguchi method-based response surface analysis". Measurement. Vol. 45, Issue 4, pp. 785-794. 2012 ISSN: 0263-2241. DOI: 10.1016/j.measurement.2011.12.004.

[19] A. Fernández Abia, J. Barreiro, L. López de Lacalle y S. Martínez Pellitero. "Behavior of austenitic stainless steels at high speed turning using specific force coefficients". The International Journal of Advanced Manufacturing Technology. Vol., pp. 1-11. 2012 ISSN: 0268-3768. DOI: 10.1007/ s00170-011-3846-9.

[20] ANSI/ASME, Tool-life testing with single-point turning tools, in B94.55M. 1985, American National Standard: New York. USA. p. 47.

[21] G. Bartarya y S.K. Choudhury. "State of the art in hard turning". International Journal of Machine Tools and Manufacture. Vol. 53, Issue 1, pp. 1-14. 2012 ISSN: 0890-6955. DOI: 10.1016/j.ijmachtools.2011.08.019.

[22] S. Rizzuti y D. Umbrello. "Prediction Of Abrasive And Diffusive Tool Wear Mechanisms In Machining”. AIP Conference Proceedings. Paris. 2011.

[23] D. Jianxin, Z. Jiantou, Z. Hui y Y. Pei. "Wear mechanisms of cemented carbide tools in dry cutting of precipitation hardening semiaustenitic stainless steels". Wear. Vol. 270, Issue 7-8, pp. 520-527. 2011 ISSN: 00431648. DOI: 10.1016/j.wear.2011.01.006.

[24] R. Suresh, S. Basavarajappa, V.N. Gaitonde y G.L. Samuel. "Machinability investigations on hardened AISI 4340 steel using coated carbide insert". International Journal of Refractory Metals and Hard Materials. Vol. 33, pp. 75-86. 2012 ISSN: 0263-4368. DOI: $10.1016 /$ j.ijrmhm.2012.02.019.

[25] I. Asiltürk y M. Çunka. "Modeling and prediction of surface roughness in turning operations using artificial neural network and multiple regression method". Expert Systems with Applications. Vol. 38, pp. 5826-5832. 2011 ISSN: 0957-4174. DOI: 10.1016/j. eswa.2010.11.041. 